\title{
Pointing accuracy of the ANTARES detector: Moon shadow and surface array analysis
}

\author{
Matteo Sanguineti* on behalf of the ANTARES collaboration \\ Università degli Studi di Genova, INFN Genova
}

E-mail: matteo.sanguinetiege.infn.it

\begin{abstract}
The ANTARES detector is the largest neutrino telescope in operation in the North Hemisphere. One of the main goals of the ANTARES telescope is the search for point-like neutrino sources. For this reason both the pointing accuracy and the angular resolution of the detector are important and a reliable way to evaluate these performances is needed. One standard method used to verify the pointing capability of a detector and to determine the instrument resolution is to observe the Moon shadowing. This corresponds to the measurement of a deficit from a narrow solid angle region centred to the Moon position due to the absorption of primary cosmic rays and a subsequent reduced flux of secondary muons. The analysis of the ANTARES data in the interval between 2008 and 2015 shows the Moon shadow with $3.5 \sigma$ significance and no evidence of a statistical significant shift from the nominal position. The results from a second, independent, study are also presented. This additional method to evaluate the pointing performance used the combined measurements of the electromagnetic component at sea level and the penetrating muons. A boat with a surface array of scintillators to detect charged particles was circled around the ANTARES telescope at various radii from its centre. The pointing performance was estimated measuring the angular correlations between the down-going showers detected by the surface array and by the muons detected underwater by the ANTARES detector. The results obtained from the two methods are consistent.
\end{abstract}

35th International Cosmic Ray Conference - ICRC2017

10-20 July, 2017

Bexco, Busan, Korea

${ }^{*}$ Speaker. 


\section{Introduction}

The ANTARES neutrino telescope [1] is the largest neutrino telescope currently in operation in the North hemisphere. It is designed for the identification of possible point-like sources of high energy cosmic neutrinos.

In the point-like source studies two of the most important characteristics of the detector are the angular resolution and the absolute pointing. These parameters have been estimated with two different approaches: the "Moon shadow" and the surface array analysis.

The detector [1] is not symmetric for upward- and downward-going particles, as the detection units (the optical modules) are looking downwards at $45^{\circ}$ in order to maximize the sensitivity for up-going neutrino-induced events. Thus, this measurement using downward-going particles represents an underestimation of the angular resolution for neutrino events.

The Moon shadow measurement is based on the research of an atmospheric muon deficit in the region around the Moon. In fact our satellite absorbs primary cosmic rays reducing the number of secondary muons produced in the atmosphere. This measurement has been performed by several collaboration: CYGNUS [2], TIBET [3], CASA [4], MACRO [5], SOUDAN [6], ARGO [7] and also IceCube [8], the other neutrino telescope currently operating at the South Pole. The results of Moon shadow analysis using the ANTARES 2008-2015 data sample are presented in Section 2.

The other approach used to estimate the pointing performance of the detector is based on the search of correlations between the down-going shower measured by a surface array of charged particle detectors located on a boat close to our neutrino telescope and ANTARES itself. Two sea campaign were performed between 2011 and 2012, the results are presented in Section 3.

\section{The Moon shadow analysis}

In this analysis atmospheric muons are used to estimate the pointing performance of the detector, while in the other ANTARES studies they represent the major background source. The primary cosmic protons are absorbed by the Moon disk, so a "shadow" of atmospheric muons should be visible (above $1 \mathrm{TeV}$ the direction of the muons is almost collinear with the primary cosmic-ray particles). Therefore measuring the event density of down-going muon tracks, the Moon is used as a "calibration source" to verify the pointing of the detector. The data are used also to estimate the angular resolution on the measurement of downward-going atmospheric muons.

The Moon shadow deficit is measured counting the number of muons detected in 25 concentric rings with increasing radius (from $0^{\circ}$ to $10^{\circ}$ with steps of $0.4^{\circ}$ ) centred on the instantaneous Moon position.

A Monte Carlo simulation has been developed with the MUPAGE code [9] in order to optimize the selection criteria of the analysis. The simulation includes also the propagation of the muons in the instrumented volume, the induced emission of Cherenkov light, the propagation of the light up to the PMTs and the detector response. The Monte Carlo takes in account also the optical background caused by bioluminescence and ${ }^{40} \mathrm{~K}$ decay.

The atmospheric muon tracks, both in the simulation and in the data sample, have been reconstructed with a robust track fitting procedure based on a maximisation likelihood method [10]. The two parameters that are used in the analysis optimization are the quality of the reconstructed track 
$\Lambda$ and the angular error of the reconstructed direction $\beta$. See further information on these variables in [11].

The Moon shadow effect is simulated rejecting the muons generated within the Moon disk, having a radius $R_{\text {Moon }}=0.26^{\circ}$. Two different Monte Carlo simulation sets were generated: one considering the shadowing effect of the Moon, rejecting the muons generated within the Moon disk, and the other without this effect.

The selection criteria optimization is performed thanks to the test statistic function $t$

$$
t=\sum_{\text {rings }} \frac{\left(n_{m}-n_{\text {exp }, N M}\right)^{2}}{n_{\text {exp }, N M}}-\frac{\left(n_{m}-n_{\text {exp }, M}\right)^{2}}{n_{\text {exp }, M}},
$$

where the sum is over all the rings around the Moon centre; $n_{m}$ is the number of events detected in a ring, $n_{\text {exp }, M}$ is the expected number of events in "Moon shadow" hypothesis and $n_{\text {exp }, N M}$ is the expected number of events in "no Moon shadow" hypothesis.

Psudo-experiments are generated using the two Monte Carlo simulations mentioned above in order to derive the distribution of the variable $t$ in the hypothesis that our experimental apparatus can observe the Moon shadow or not. The test statistic allow to find cuts on quality parameters $\Lambda$ and $\beta$ yielding the best event selection for this analysis: track quality estimator $\Lambda>-5.9$ and angular error $\beta<0.8^{\circ}$. The distribution of the test statistics obtained with the mentioned values for the quality parameter cuts and the two alternative hypotheses are presented in Fig. 1.

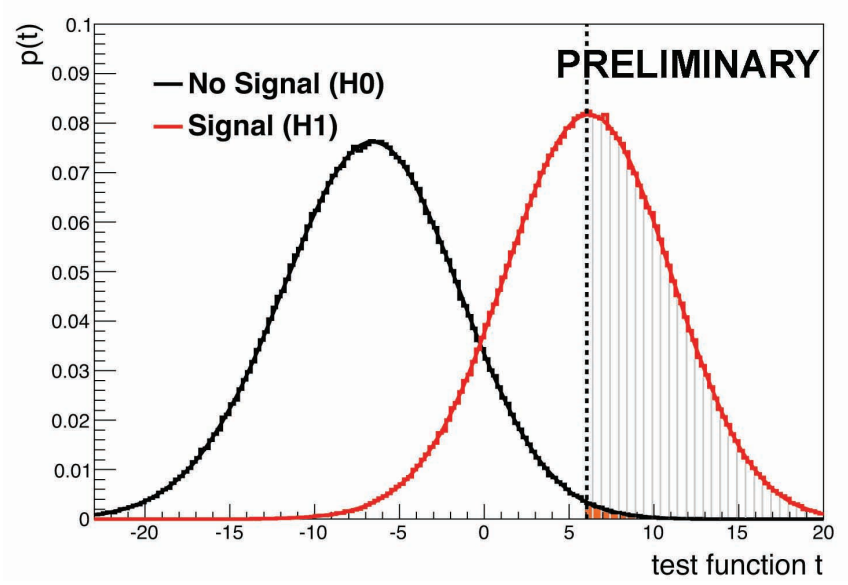

Figure 1: The test function $t$ distribution for "Moon shadow" hypothesis (red curve) and "no Moon shadow" hypothesis (black curve). The orange area quantifies the probability that the Moon shadow effect is not observed if it actually occurs $(3.4 \sigma)$. The shaded area is the fraction of the toy experiments where the Moon shadow hypothesis is correctly identified as evidence of the shadowing effect $(50 \%)$.

The events in the 2008-2015 ANTARES data sample have been selected with the optimized cut described above and the muon density close to the Moon region is derived. Events are binned using concentric rings around the Moon centre up to an angular distance of $10^{\circ}$ (bin size of $0.4^{\circ}$ ). The muon density is presented in Fig. 2 .

The muon shadowing is clear close to the nominal Moon region. The angular resolution of the detector is evaluated by fitting the distribution in Fig. 2 with the following function [6] 


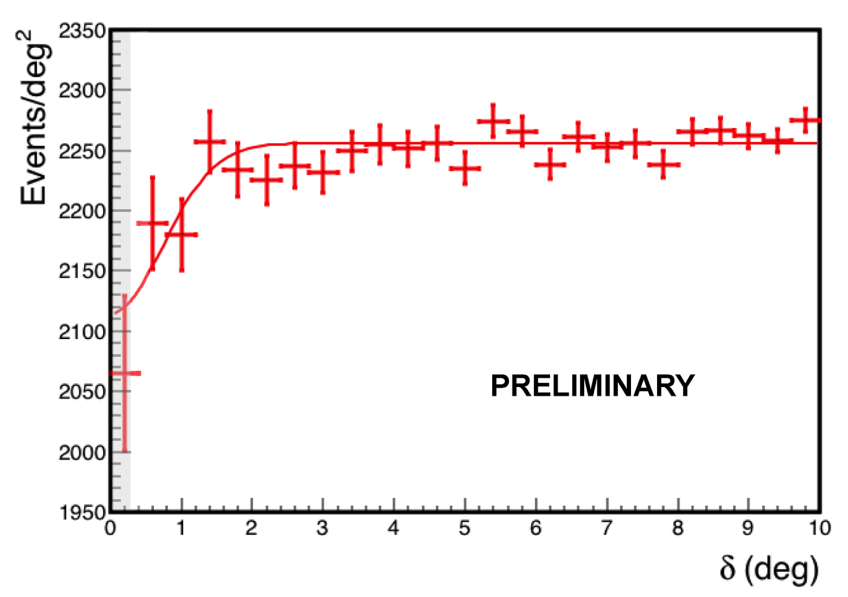

Figure 2: The muon events density as a function of the angular distance from the Moon centre. The shaded area corresponds to the apparent radius of the Moon $\left(0.26^{\circ}\right)$.

$$
\frac{d n}{d \delta^{2}}=k\left(1-\frac{R_{M o o n}^{2}}{2 \sigma^{2}} e^{-\frac{\delta^{2}}{2 \sigma^{2}}}\right)
$$

where $k$ us the average muon event density in the "no Moon shadow" scenario, $\sigma$ is the detector angular resolution for atmospheric down-going muons, $R_{M o o n}$ is the Moon radius $\left(0.26^{\circ}\right)$ and $\delta$ is the angular distance of the muons events from the Moon.

The measure of the angular resolution for down-going atmospheric muons resulting from the fit is $0.73^{\circ} \pm 0.15^{\circ}$. The significance of the shadowing has been evaluated using a $\chi^{2}$ test on the data of Fig. 2 assuming the "no Moon shadow" scenario as null hypothesis. The corresponding significance of Moon shadowing is $3.5 \sigma$.

The absolute pointing of the detector has been evaluated with a method inspired by the approach used in [5]. A $10^{\circ} \times 10^{\circ}$ grid of square bins (bin width $=0.2^{\circ}$ ) with the centre of the grid coincident with the Moon nominal position has been considered and a test statistic function $\Delta \chi^{2}$ has been defined

$$
\Delta \chi^{2}\left(x_{s}, y_{s}\right)=\chi_{M}^{2}\left(x_{s}, y_{s}, S_{M}\right)-\chi_{N M}^{2}(0)
$$

where $\chi_{N M}^{2}$ is the $\chi^{2}$ value assuming no shadowing effect, $\chi_{M}^{2}$ is the $\chi^{2}$ value assuming that the shadowing occurs, $\left(x_{s}, y_{s}\right)$ is the value of the assumed pointing shift and $S_{M}$ is the shadowing effect strength. In the case of no shadowing the strength $S_{M}$ is equal to 0 .

The map of $\Delta \chi^{2}$ for different assumed pointing shift is presented in Fig. 3. The corresponding evaluation of confidence level of the Moon shadow pointing accuracy is shown in Fig. 4. The nominal position of the Moon $(0,0)$ is compatible with a null shift at $1.3 \sigma$. 


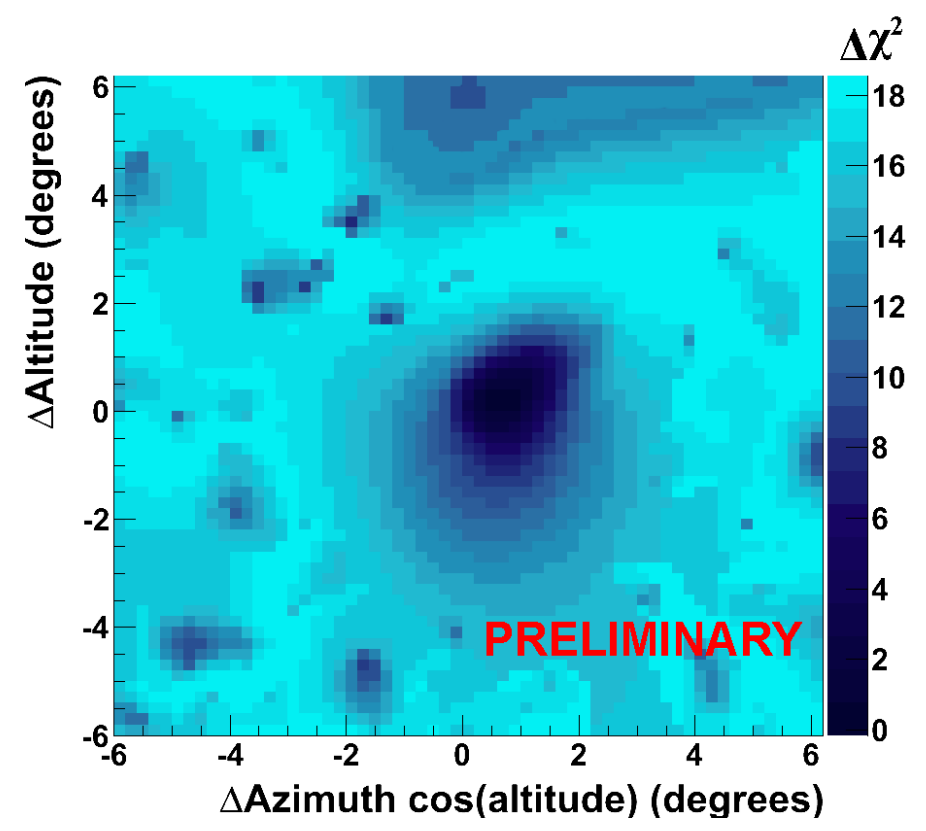

Figure 3: Map of $\Delta \chi^{2}$ as a function of the assumed pointing shift.

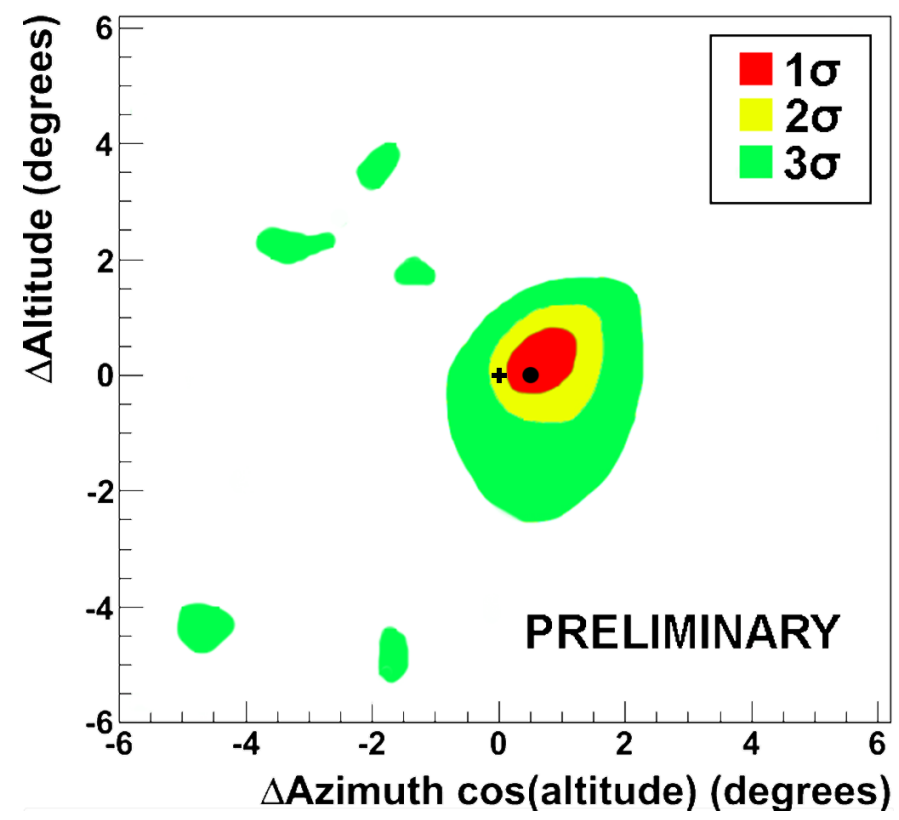

Figure 4: Contour plot of the Moon shadow pointing accuracy (red: 68\% contour; yellow: 95\% contour; green: $99 \%$ contour). The black dot represents the position of the most probable pointing shift. 


\section{Surface array analysis}

Two sea campaign have been performed by the ANTARES collaboration in 2011 and 2012 where 15 liquid scintillator detection units have been located on a boat circulating around the site of the ANTARES detector. This approach allows to estimate the detector pointing performance using the coincidences between the scintillators and the telescope below.

The direction of the coincidence events was reconstructed using the known position of the boat (thanks to a GPS system) and the detector. The selection requirement for the events detected by the surface array is a coincidence in at least 3 detection units in a time window of $650 \mathrm{~ns}$. This selection leads to a trigger rate of $1 \mathrm{~Hz}$. On the other hand the selection criteria of the ANTARES reconstructed events in the corresponding time window are $\Lambda>-6$ and $\beta>0.6^{\circ}$ (rate around 0.25 $\mathrm{Hz})$.
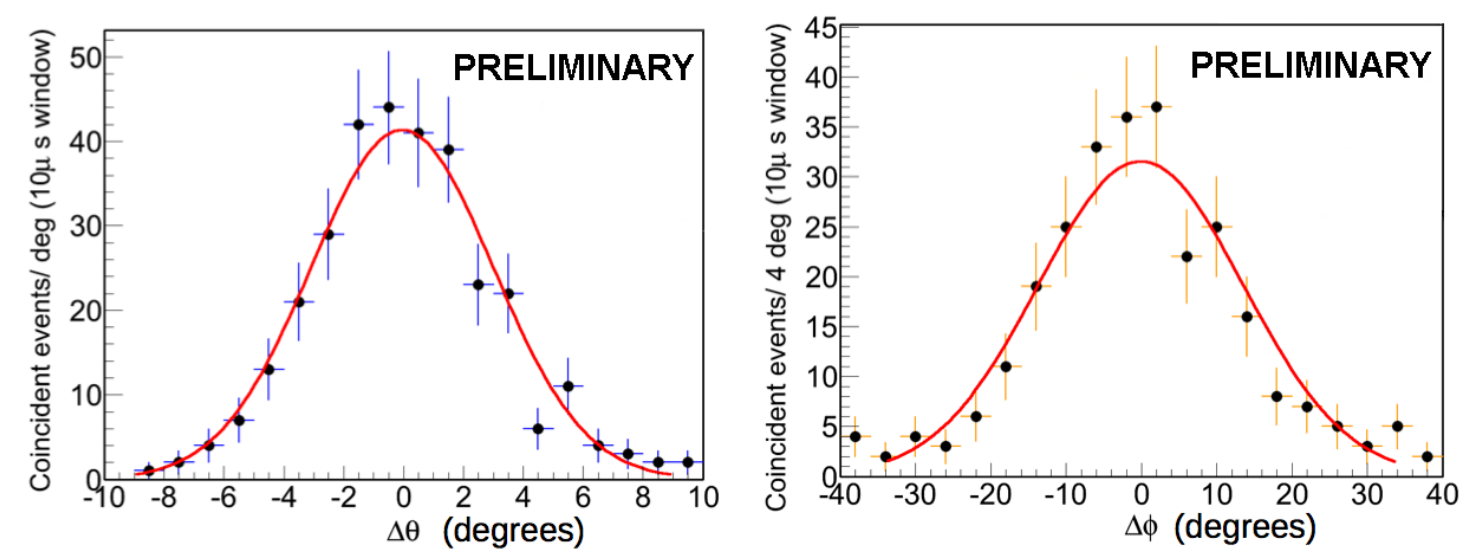

Figure 5: Difference between the shower angle evaluated with ANTARES reconstruction and the shower angle evaluated with the surface array system (using the relative position of the boat and ANTARES). Difference for zenith angle $\theta$ (left) and azimuth angle $\phi$ (right).

The results obtained in the two campaigns are presented in Fig. 5: The distribution of the difference between the shower angle evaluated with ANTARES reconstruction and the shower angle evaluated with the surface array system (using the relative position of the boat and ANTARES) allows to estimate the pointing performance of the detector. The surface array analysis is consistent with a correct alignment of the detector. This result is compatible with constraints derived with the Moon shadow analysis.

\section{Conclusions}

The absolute pointing of the ANTARES detector have been estimated exploiting the Moon shadow effect and a surface array system. The measurement has allowed also the estimation of the detector angular resolution for the measurement of atmospheric muons.

The 2007-2015 ANTARES data sample shows a 3.5 $\sigma$ evidence of Moon shadow effect and corresponding estimation of the detector angular resolution for atmospheric down-going muons is $0.73^{\circ} \pm 0.15^{\circ}$. The ANTARES pointing accuracy does not present any evident pointing shifts. 
The Moon shadow results have been also confirmed by the surface array campaign performed between 2011 and 2012. The surface array analysis is consistent with a correct alignment of the detector.

\section{References}

[1] M. Ageron et al. (ANTARES Coll.), Nuclear Instruments \& Methods in Physics Research A656, 11-38 (2011)

[2] D. E. Alexandreas et al., Phy. Rev., D43:1735-1738 (1991)

[3] M. Amenomori et al., Phys. Rev., D47:2675-2681 (1993).

[4] A. Borione et al. , Phys. Rev., D49:1171-1177 (1994).

[5] M. Ambrosio et al., Phys. Rev., D59:012003 (1999).

[6] H. Cobbs et al. , Phys. Rev., D61:092002 (2000).

[7] ARGO-YBJ Collaboration, Phys. Rev., D.84:022003 (2011).

[8] ICECUBE collaboration, Phys. Rev. D 89, 102004 (2014).

[9] G. Carminati, A. Margiotta, M. Spurio Comput. Phys. Commun. 179, 915-923 (2008)

[10] S. Adrian-Martinez et al. (ANTARES Coll.), JCAP 1303, 006 (2013)

[11] A. Albert et al. (ANTARES Coll.), arXiv:1706.01857 (2017) 